УДК 517.54

F. Nuray, R. F. Patterson

\title{
VECTOR-VALUED BIVARIATE ENTIRE FUNCTIONS OF BOUNDED INDEX SATISFYING A SYSTEM OF DIFFERENTIAL EQUATIONS
}

F. Nuray, R. F. Patterson. Vector-valued bivariate entire functions of bounded index satisfying a system of differential equations, Mat. Stud. 49 (2018), 67-74.

The concept of complex valued bivariate entire functions of bounded index is extended to $\mathbb{C}^{n}$ valued bivariate entire functions by replacing the absolute value in the definition of an entire function of bounded index by the maximum of the absolute values of the components. If the components of a $\mathbb{C}^{n}$-valued bivariate entire function are of bounded index, then the function is also of bounded index. We present sufficient conditions providing index boundedness of bivariate vector-valued entire solutions of certain system of partial differential equations.

1. Introduction. If $f(z, w)$ is a bivariate entire function then at a point $(a, b) \in \mathbb{C}^{2}$ the function $f(z, w)$ has a bivariate Taylor expansion

$$
f(z, w)=\sum_{k=0}^{\infty} \sum_{l=0}^{\infty} c_{k l}(z-a)^{k}(w-b)^{l},
$$

where

$$
c_{k l}=\frac{1}{k ! l !}\left[\frac{\partial^{k+l} f(z, w)}{\partial w^{k} \partial z^{l}}\right]_{z=a ; w=b}=\frac{1}{k ! l !} f^{(k, l)}(a, b) .
$$

If the bivariate series is absolutely convergent then $\lim _{k, l}\left|c_{k l}\right|=0$.

Similar to Gross ([6]) we presented in [9-11] the following notion of bivariate entire function of bounded index.

Definition 1. A bivariate entire function $f$ is said to be of bounded index provided that there exist integers $M$ and $N$ independent of $z$ and $w$ such that

$$
\max _{0 \leq k \leq M ; 0 \leq l \leq N}\left\{\frac{\left|f^{(k, l)}(z, w)\right|}{k ! l !}\right\} \geq \frac{\left|f^{(i, j)}(z, w)\right|}{i ! j !}
$$

for all $i$ and $j$.

We shall say that $f$ is of index $(M, N)$ if $N$ ad $M$ are the smallest integers for which above inequality holds. But it leads to the following question: What is the index of the function of $f$ if the corresponding inequality holds for $(N, M)$ and $(M, N)$ ? Thus, the index of the function is not uniquely defined. It is better to define an index of a bivariate function as

2010 Mathematics Subject Classification: 30D10, 30D20.

Keywords: entire function; bivariate function; bounded index.

doi:10.15330/ms.49.1.67-74

(C) F. Nuray, R. F. Patterson, 2018 
height of the vector $(N, M)$ i.e. $N+M$. The least such integer $N+M$ is called the index of the function $f$ and is denoted by $N(f)$. A bivariate entire function which is not of bounded index is said to be of unbounded index. The primary definition of entire function of several variables of bounded index was introduced in [1]. Next, for simplicity the case of bivariate entire functions is considered in [14,15].

In this paper, we are concerned with one possible extension of this concept to vectorvalued bivariate functions. Let $A^{T}$ be a transpose of a $n \times n$ matrix $A$. If $f_{i}(z, w) i=$ $1,2, \ldots, n$, are complex-valued bivariate entire functions, then

$$
F(z, w)=\left(f_{1}(z, w), f_{2}(z, w), \ldots, f_{n}(z, w)\right)^{T},
$$

is a $\mathbb{C}^{n}$-valued bivariate entire vector function and we write

$$
\|F(z, w)\|=\max _{1 \leq i \leq n}\left\{\left|f_{i}(z, w)\right|\right\}
$$

and for $i, j \in \mathbb{Z}_{+}$

$$
F^{(i, j)}(z, w)=\frac{\partial^{i+j} F(z, w)}{\partial w^{i} \partial z^{j}}=\left(\frac{\partial^{i+j} f_{1}(z, w)}{\partial w^{i} \partial z^{j}}, \frac{\partial^{i+j} f_{2}(z, w)}{\partial w^{i} \partial z^{j}}, \ldots, \frac{\partial^{i+j} f_{n}(z, w)}{\partial w^{i} \partial z^{j}}\right)^{T} .
$$

\section{Main Result.}

Definition 2. A vector valued bivariate entire function $F(z, w)$ is said to be of bounded index provided that there exist integers $M$ and $N$ independent of $z$ and $w$ such that

$$
\max _{0 \leq k \leq M ; 0 \leq l \leq N}\left\{\frac{\left\|F^{(k, l)}(z, w)\right\|}{k ! l !}\right\} \geq \frac{\left\|F^{(i, j)}(z, w)\right\|}{i ! j !}
$$

for all $i$ and $j$.

The least such integer $N+M$ is called the index of the vector function $F$ and is denoted by $N(F)$.

A concept of bounded index for vector-valued entire function of one variable (i.e. an entire curve) is considered in $[7,12,13]$. Particularly, they presented examples of entire curves of bounded index without all of their components being of bounded index. Also there are two papers about analytic curves of bounded $l$-index $([5,16])$. The mentioned papers are devoted to analytic theory of systems of ordinary differential equations. But we will study entire functions $F: \mathbb{C}^{2} \rightarrow \mathbb{C}^{n}$ and our definition of bounded index uses partial derivatives as in recent papers about $\mathbf{L}$-index in joint variables $([2,3])$. Note that there is another multidimensional approach using directional derivatives in the definition so called functions of bounded L-index in direction ([4]). It is possible to use various norms in inequality (1). Particularly, instead the sup-norm we can consider the Euclidean norm. But M. Bordulyak and M. Sherementa ([5]) proved that an analytic curve $F: \mathbb{D}_{R} \rightarrow C^{n}$ is of bounded lindex by the sup-norm if and only if $F$ is of bounded $l$-index by the Euclidean norm, where $\mathbb{D}_{R}=\{z \in C:|z|<R\}, R \in(0, \infty]$. Therefore, we will consider only the sup-norm.

Lemma 1. If $f_{1}(z, w), f_{2}(z, w), \ldots, f_{n}(z, w)$ are bivariate entire functions of bounded index with indexes $N\left(f_{1}\right)=N_{1}, N\left(f_{2}\right)=N_{2}, \ldots, N\left(f_{n}\right)=N_{n}$ respectively, then

$$
F(z, w)=\left(f_{1}(z, w), f_{2}(z, w), \ldots, f_{n}(z, w)\right)
$$

is of bounded index $M$ such that $M \leq N=\max \left\{N_{1}, N_{2}, \ldots, N_{n}\right\}$. 
Proof. For $p \in\{1,2, \ldots, n\}$, we write

$$
\begin{gathered}
\frac{\left|f_{p}^{(i, j)}(z, w)\right|}{i ! j !} \leq \max _{0 \leq k+l \leq N_{p}}\left\{\frac{\left|f_{p}^{(k, l)}(z, w)\right|}{k ! l !}\right\}= \\
\max _{0 \leq k+l \leq N}\left\{\frac{\left|f_{p}^{(k, l)}(z, w)\right|}{k ! l !}\right\} \leq \max _{0 \leq k+l \leq N}\left\{\frac{\left\|F^{(k, l)}(z, w)\right\|}{k ! l !}\right\} .
\end{gathered}
$$

Therefore, (1) is satisfied.

If $A=\left[a_{i j}\right]$ is an $n \times n$ matrix, we use the norm

$$
\|A\|=\max _{1 \leq i \leq n} \sum_{j=1}^{n}\left|a_{i j}\right|
$$

Theorem 1. If a vector valued bivariate entire function $F: \mathbb{C}^{2} \rightarrow \mathbb{C}^{n}$ is a solution of the following system of partial differential equations

$$
F^{(1,0)}(z, w)=A_{10} F(z, w), \quad F^{(0,1)}(z, w)=A_{01} F(z, w),
$$

where $A_{10}, A_{01}$ are constant $n \times n$ matrices, then $F(z, w)$ is an entire function of bounded index with index $N(F) \leq P$ where

$$
P=p_{1}+q_{1}, \quad p_{1}=\min \left\{p \in \mathbb{Z}_{+}:\left\|A_{10}\right\| \leq(p+1)\right\}, \quad q_{1}=\min \left\{p \in \mathbb{Z}_{+}:\left\|A_{01}\right\| \leq(q+1)\right\} .
$$

Proof. For all integers $p \geq 1$ and $q \geq 1$ and all $(z, w) \in \mathbb{C}^{2}$ we have

$$
F^{\left(p_{1}+p, q\right)}(z, w)=A_{10} F^{\left(p_{1}+p-1, q\right)}(z, w), \quad F^{\left(p, q_{1}+q\right)}(z, w)=A_{01} F^{\left(p, q_{1}+q-1\right)}(z, w)
$$

Therefore

$$
\begin{aligned}
& \frac{\left\|F^{\left(p_{1}+p, q\right)}(z, w)\right\|}{\left(p_{1}+p\right) ! q !} \leq \frac{\left\|A_{10}\right\|}{\left(p_{1}+p\right)} \frac{\left\|F^{\left(p_{1}+p-1, q\right)}(z, w)\right\|}{\left(p_{1}+p-1\right) ! q !} \\
& \frac{\left\|F^{\left(p, q_{1}+q\right)}(z, w)\right\|}{p !\left(q_{1}+q\right) !} \leq \frac{\left\|A_{01}\right\|}{\left(q_{1}+q\right)} \frac{\left\|F^{\left(p, q_{1}+q-1\right)}(z, w)\right\|}{p !(q+1+q-1) !}
\end{aligned}
$$

and so

$$
\frac{\left\|F^{(i, j)}(z, w)\right\|}{i ! j !} \leq \max _{0 \leq k+l \leq p_{1}+q_{1}}\left\{\frac{\left\|F^{(k, l)}(z, w)\right\|}{k ! l !}\right\}
$$

for all $i=0,1,2, \ldots$ and $j=0,1,2, \ldots$ and all $(z, w) \in \mathbb{C}^{2}$.

In the proof of Theorem 2 we need the following lemma.

Lemma 2 ([14,15]). An entire bivariate function $f$ is of bounded index if and only if there exist integers $M>0, N>0$ and a constant $C>0$ such that

$$
\max _{0 \leq i \leq M, 0 \leq j \leq N}\left\{\left|f^{(M+1, i)}(z, w)\right|,\left|f^{(j, N+1)}(z, w)\right|\right\} \leq C \max _{0 \leq i \leq M ; 0 \leq j \leq N}\left\{\left|f^{(i, j)}(z, w)\right|\right\}
$$

for all $(z, w) \in \mathbb{C}^{2}$. 
Theorem 2. Let $f(z, w)$ be a transcendental entire bivariate function and satisfying the system of partial differential equations

$$
\begin{aligned}
& P_{m 0}(z, w) f^{(m, 0)}(z, w)+\sum_{i=0}^{m-1} \sum_{j=0}^{n-1} P_{i j}(z, w) f^{(i, j)}(z, w)=g(z, w), \\
& Q_{0 n}(z, w) f^{(0, n)}(z, w)+\sum_{i=0}^{m-1} \sum_{j=0}^{n-1} Q_{i j}(z, w) f^{(i, j)}(z, w)=h(z, w),
\end{aligned}
$$

where $P_{i j}(z, w), Q_{i j}(z, w), i=0,1,2, \ldots, m ; j=0,1, \ldots, n$ are polynomials, $P_{m 0}(z, w)$ and $Q_{0 n}(z, w)$ are polynomials with separable variables of degree in each variable not less than degree of any $P_{i j}(z, w)$ and $Q_{i j}(z, w)$ in the same variable accordingly and $g(z, w), h(z, w)$ are entire functions of bounded index. Then $f(z, w)$ is of bounded index.

Proof. If $g(z, w)$ is of bounded index then there exist integers $M \geq 0$ and $N \geq 0$ and constant $C>0$ such that

$$
\max _{0 \leq i \leq M, 0 \leq j \leq N}\left\{\left|g^{(M+1, i)}(z, w)\right|,\left|g^{(j, N+1)}(z, w)\right|\right\} \leq C \max _{0 \leq i \leq M ; 0 \leq j \leq N}\left\{\left|g^{(i, j)}(z, w)\right|\right\}
$$

for all $(z, w) \in \mathbb{C}^{2}$. For convenience we assume that $P_{i, n}(z, w) \equiv 0$ and $P_{m, j}(z, w) \equiv 0$ for $i$, $j \in \mathbb{N}$. Thus

$$
\begin{gathered}
\left|g^{(M+1, i)}(z, w)\right|=\left|\sum_{k=0}^{m} \sum_{j=0}^{n} \frac{\partial^{M+i+1}}{\partial z^{M+1} \partial w^{i}} P_{k j}(z, w) f^{(k, j)}(z, w)\right|= \\
=\left|\sum_{k=0}^{m} \sum_{j=0}^{n} \sum_{t=0}^{M+1} \sum_{l=0}^{i}\left(\begin{array}{c}
M+1 \\
t
\end{array}\right)\left(\begin{array}{c}
i \\
l
\end{array}\right) P_{k j}^{(t, l)}(z, w) f^{(k+M+1-t, j+i-l)}(z, w)\right| \leq \\
\leq C \max _{0 \leq k \leq M ; 0 \leq j \leq n}\left\{\left|g^{(k, j)}(z, w)\right|\right\} .
\end{gathered}
$$

Hence

$$
\begin{gathered}
\left|P_{00}(z, w) f^{(m+M+1, i)}(z, w)\right| \leq \\
\leq \mid \sum_{k=0}^{m} \sum_{j=0}^{n} \sum_{t=0}^{M+1} \sum_{l=0}^{i}\left(\begin{array}{c}
M+1 \\
t
\end{array}\right)\left(\begin{array}{c}
i \\
l
\end{array}\right) P_{k j}^{(t, l)}(z, w) f^{(k+M+1-t, j+i-l)}(z, w) \\
+\sum_{t=1}^{M+1} \sum_{l=1}^{i}\left(\begin{array}{c}
M+1 \\
t
\end{array}\right)\left(\begin{array}{c}
i \\
l
\end{array}\right) P_{m 0}^{(t, l)}(z, w) f^{(m+M+1-t, n+i-l)}(z, w) \mid+ \\
+C \max _{0 \leq k \leq M ; 0 \leq j \leq N}\left\{\left|\sum_{t=0}^{m} \sum_{l=0}^{n} \sum_{r=0}^{k} \sum_{s=0}^{j}\left(\begin{array}{c}
k \\
r
\end{array}\right)\left(\begin{array}{c}
j \\
s
\end{array}\right) P_{t l}^{(r, s)}(z, w) f^{(t+k-r, l+j-s)}(z, w)\right|\right\} \leq \\
\leq(C+1)(m+1)(n+1)(M+2)(M+1) !(N+2)(N+1) ! \times \\
\times \max _{0 \leq k \leq m ; 0 \leq j \leq n ; 0 \leq t \leq M+1 ; 0 \leq l \leq i}\left\{\left|P_{k j}^{(t, l)}(z, w)\right|\right\}
\end{gathered}
$$

Now since degree of $P_{00}$ is greater than degree of $P_{k j}$ in each variable, $k=0 ., 1,2, \ldots, m$; $j=0,1,2, \ldots, n$, there exist $K>0, R>0$ and $Q>0$ such that

$$
\left|P_{m 0}(z, w)\right| \geq K\left|P_{k j}^{(t, l)}(z, w)\right| k=0,1,2, \ldots, M+1 \text { and } l=0,1,2, \ldots, i
$$


and for all $|z| \geq R$ and $|w| \geq Q$. Thus,

$$
\begin{gathered}
\left|f^{(m+M+1, n+i)}(z, w)\right| \leq \\
\leq(C+1)(m+1)(n+1)(M+2)(M+1) !(i+1) i ! \max _{0 \leq k \leq m+M ; 0 \leq j \leq i-1+n}\left\{\left|f^{(k, j)}(z, w)\right|\right\}
\end{gathered}
$$

for $|z|>R$ and $|w|>Q$. Hence, for

$$
\begin{gathered}
S_{1}=(C+1)(m+1)(n+1)(M+2)(M+1) !(i+1) i !, T=m+M, U=n+i, \\
\left|f^{(T+1, U)}(z, w)\right| \leq S_{1} \max _{0 \leq k \leq T ; 0 \leq j \leq U}\left\{\left|f^{(k, j)}(z, w)\right|\right\}
\end{gathered}
$$

for $|z| \geq R$ and $|w| \geq Q$. Similarly, it can be proved that

$$
\left|f^{(T, U+1)}(z, w)\right| \leq S_{2} \max _{0 \leq k \leq T ; 0 \leq j \leq U}\left\{\left|f^{(k, j)}(z, w)\right|\right\} .
$$

These inequalities hold for all $|z|<R$ and $|w|<Q$. Choosing $S \geq \max \left\{S_{1}, S_{2}\right\}$ by Lemma 2, we have $f$ is bounded index.

Theorem 3. If a vector valued bivariate entire function $F: \mathbb{C}^{2} \rightarrow \mathbb{C}^{n}$ is a solution of system of partial differential equations

$$
\begin{aligned}
& P_{m 0}(z, w) F^{(m, 0)}(z, w)+\sum_{i=0}^{m-1} \sum_{j=0}^{n-1} P_{i j}(z, w) F^{(i, j)}(z, w)=g(z, w), \\
& Q_{0 n}(z, w) F^{(0, n)}(z, w)+\sum_{i=0}^{m-1} \sum_{j=0}^{n-1} Q_{i j}(z, w) F^{(i, j)}(z, w)=h(z, w),
\end{aligned}
$$

where $P_{i j}(z, w), Q_{i j}(z, w), i=0,1,2, \ldots, m ; j=0,1, \ldots, n$ are polynomials and $P_{m 0}(z, w)$, $Q_{0 n}(z, w)$ are polynomials with separable variables of degree in each variable not less than that of degree of any $P_{i j}(z, w)$ and $Q_{i j}(z, w)$ in each variable respectively and $g(z, w), h(z, w)$ are entire functions of bounded index, then each component of $F(z, w)$ is of bounded index.

Proof. Since each component of $F(z, w)$ satisfies a system of partial differential equations of the form in Theorem 2, each component is of bounded index.

Theorem 4. If a vector valued bivariate entire function $F: \mathbb{C}^{2} \rightarrow \mathbb{C}^{n}$ is a solution of the following system of partial differential equation

$$
F^{(1,0)}(z, w)=A_{10} F(z, w)+R_{1}(z, w), \quad F^{(0,1)}(z, w)=A_{01} F(z, w)+R_{2}(z, w),
$$

where $A_{10}, A_{01}$ are constant $n \times n$ matrices, then $F(z, w)$ is an entire function of bounded index with index $N(F) \leq P$ where

$$
\begin{gathered}
P=p_{1}+q_{1}, \quad p_{1}=\min \left\{p \in \mathbb{Z}_{+}:\left\|A_{10}\right\| \leq(p+1) \text { and } p-1 \geq \max _{1 \leq i \leq n} \operatorname{deg} r_{i 1}\right\}, \\
q_{1}=\min \left\{q \in \mathbb{Z}_{+}:\left\|A_{01}\right\| \leq(q+1) \text { and } q-1 \geq \max _{1 \leq i \leq n} \operatorname{deg} r_{i 2}\right\}, \\
R_{1}(z, w)=\left(r_{11}(z, w), r_{21}(z, w), \ldots, r_{n 1}(z, w)\right), \\
R_{2}(z, w)=\left(r_{12}(z, w), r_{22}(z, w), \ldots, r_{n 2}(z, w)\right)
\end{gathered}
$$

are vector valued polynomials. 
Proof. Let $p$ and $q$ be two nonnegative integers such that $\left\|A_{10}\right\| \leq(p+1)$ and $p-1 \geq$ $\max _{1 \leq i \leq n} \operatorname{deg} r_{i 1},\left\|A_{01}\right\| \leq(q+1)$ and $q-1 \geq \max _{1 \leq i \leq n} \operatorname{deg} r_{i 2}$. Then

$$
F^{(m+p, n)}(z, w)=A_{10} F^{(m+p-1, n)}(z, w)+R^{(m+p-1, n)}(z, w)=A_{10} F^{(m+p-1, n)}(z, w)
$$

for all integers $m \geq 1$ and $n \geq 1$ and all $(z, w) \in \mathbb{C}^{2}$. Therefore,

$$
\frac{\left\|F^{(m+p, n)}(z, w)\right\|}{(m+p) ! n !} \leq \frac{\left\|A_{10}\right\|}{(m+p)} \frac{\left\|F^{(m+p-1, n)}(z, w)\right\|}{(m+p-1) ! n !}
$$

similarly

$$
\frac{\left\|F^{(m, n+q)}(z, w)\right\|}{m !(n+q) !} \leq \frac{\left\|A_{01}\right\|}{(n+q)} \frac{\left\|F^{(m, n+q-1)}(z, w)\right\|}{m !(n+q-1) !}
$$

and so

$$
\frac{\left\|F^{(i, j)}(z, w)\right\|}{i ! j !} \leq \max _{0 \leq k+l \leq p+q}\left\{\frac{\left\|F^{(k, l)}(z, w)\right\|}{k ! l !}\right\}
$$

for all $i \in \mathbb{Z}_{+}, j \in \mathbb{Z}_{+}$and all $(z, w) \in \mathbb{C}^{2}$.

Theorem 5. If $F: \mathbb{C}^{2} \rightarrow \mathbb{C}^{n}$ is an entire solution of the following system of partial differential equations

$$
F^{(1,0)}(z, w)=A_{10} F(z, w)+R_{1}(z, w), \quad F^{(0,1)}(z, w)=A_{01} F(z, w)+R_{2}(z . w),
$$

where $A_{10}, A_{01}$ are $n \times n$ matrices whose entries are rational bivariate functions which are bounded at infinity and $R_{1}(z, w), R_{2}(z, w)$ are vector valued functions whose components are rational functions which are bounded at infinity, then $F(z, w)$ is a function of bounded index.

Proof. Let $p_{0 i}$ be the least common denominator of the $a_{i j}$ 's and $r_{i}$ 's where $R_{1}=\left(r_{11}, r_{21}, \ldots\right.$, $\left.r_{n 1}\right), a_{i j}$ are entries of $A_{10}$. Let $b_{i j}=p_{0 i} s_{i j}$ and $p_{i}=p_{0 i} r_{i}$. Then $b_{i j}$ and $p_{i}$ are bivariate polynomials of degree $\leq \operatorname{deg} p_{0 i}$. Let $h=B_{10}=\left[b_{i j}\right]$ and $P=\left(p_{1}, p_{2}, \ldots, p_{n}\right)^{T}, P_{0}=$ $\left(p_{01}, \ldots, p_{0 n}\right)$ and so $F$ is a solution of $F^{(1,0)}(z, w) P_{0}=B_{11} F(z, w)+P$. Differentiating $M$ times with respect to $z$ and $N$ times with respect to $w$ where $M \geq h+1$ and $N \geq h+1$, we obtain

$$
\begin{aligned}
& \sum_{k=0}^{M} \sum_{l=0}^{N}\left(\begin{array}{c}
M \\
k
\end{array}\right)\left(\begin{array}{c}
N \\
l
\end{array}\right) p_{0}^{(k, l)} F^{(M+1-k, N-l)}(z, w)= \\
= & \sum_{k=0}^{M} \sum_{l=0}^{N}\left(\begin{array}{c}
M \\
k
\end{array}\right)\left(\begin{array}{c}
N \\
l
\end{array}\right) B_{11}^{(k, l)}(z, w) F^{(M-k, N-l)}(z, w)
\end{aligned}
$$

which simplifies

$$
\begin{gathered}
p_{0} F^{(M+1, N)}(z, w)=\sum_{k=0}^{h} \sum_{l=0}^{h}\left(\begin{array}{c}
M \\
k
\end{array}\right)\left(\begin{array}{c}
N \\
l
\end{array}\right) B_{11}^{(k, l)}(z, w) F^{(M-k, N-l)}(z, w)- \\
-\sum_{k=1}^{h} \sum_{l=1}^{h}\left(\begin{array}{c}
M \\
k
\end{array}\right)\left(\begin{array}{c}
N \\
l
\end{array}\right) p_{0}^{(k, l)}(z, w) F^{(M+1-k, N-l)}(z, w)
\end{gathered}
$$


because $\operatorname{deg} b_{i j} \leq h$. Therefore

$$
\begin{gathered}
\frac{F^{(M+1, N)}(z, w)}{(M+1) ! N !}=\frac{1}{(M+1)} \sum_{k=0}^{h} \sum_{l=0}^{h} \frac{1}{k ! l !} \frac{B_{11}^{(k, l)}(z, w)}{p_{0}} \frac{F^{(M-k, N-l)}(z, w)}{(M-k) !(N-l) !}- \\
-\sum_{k=1}^{h} \sum_{l=1}^{h} \frac{1}{k ! l !}\left(1-\frac{k}{M+1}\right)\left(1-\frac{l}{N}\right) \frac{p_{0}^{(k, l)}(z, w)}{p_{0}(z, w)} \frac{F^{(M+1-k, N+1-l)}(z, w)}{(M+1-k) !(N-l) !} .
\end{gathered}
$$

Since

$$
\frac{p_{0}^{(k, l)}(z, w)}{p_{0}} \rightarrow 0 \quad|z| \rightarrow \infty, \quad|w| \rightarrow \infty \quad \text { for } \quad k=1,2, \ldots, h ; l=1,2, \ldots, h
$$

there exists $T>0$ such that

$$
\sum_{k=1}^{h} \sum_{l=1}^{h}\left|\frac{p_{0}^{(k, l)}}{p_{0}}\right|<\frac{1}{4} \quad \text { if } \quad|z| \geq T, \quad|w| \geq T .
$$

The inequality $\operatorname{deg} b_{i j} \leq h$ yields

$$
\sum_{k=0}^{h} \sum_{l=0}^{h} \frac{1}{k ! l !} \frac{\left\|B_{11}^{(k, l)}(z, w)\right\|}{\left|p_{0}(z, w)\right|} \leq H
$$

if $|z| \geq T,|w| \geq T$, where $H$ is a constant. Choose $M_{1} \geq h+1$ and $N_{1} \geq h+1$ such that $\frac{1}{\left(M_{1}+1\right)} H<\frac{1}{4}$. Then

$$
\frac{1}{(M+1)} \sum_{k=0}^{h} \sum_{l=0}^{h} \frac{1}{k ! l !} \frac{\left\|B_{11}^{(k, l)}(z, w)\right\|}{\left|p_{0}(z, w)\right|}<\frac{1}{4}
$$

if $M \geq M_{1}, N \geq N_{1}$, and $|z| \geq T,|w| \geq T$. If $M \geq M_{1}, N \geq N_{1}$, and $|z| \geq T,|w| \geq T$, we have

$$
\begin{aligned}
\frac{\left\|F^{(M+1, N)}(z, w)\right\|}{(M+1) ! N !} \leq & {\left[\frac{1}{(M+1)} \sum_{k=0}^{h} \sum_{l=0}^{h} \frac{1}{k ! l !} \frac{\left\|B_{11}^{(k, l)}(z, w)\right\|}{p_{0}(z, w)}+\sum_{k=1}^{h} \sum_{l=1}^{h} \frac{1}{k ! l !}\left|\frac{p_{0}^{(k, l)}(z, w)}{p_{0}}\right|\right] \times } \\
& \times \max _{0 \leq i \leq M_{1} ; 0 \leq j \leq N_{1}} \frac{\left\|F^{(i, j)}\right\|}{i ! j !} .
\end{aligned}
$$

Therefore

$$
\frac{\left\|F^{(M+1, N)}(z, w)\right\|}{(M+1) ! N !} \leq \max _{0 \leq i \leq M ; 0 \leq j \leq N} \frac{\left\|F^{(i, j)}(z, w)\right\|}{i ! j !}
$$

for all $M \geq M_{1}, N \geq N_{1}$ and $|z| \geq T,|w| \geq T$. But every entire function is of bounded index on a compact set. Thus, there are integers $M_{2}$ and $N_{2}$ such that

$$
\frac{\left|f^{(i, j)}(z, w)\right|}{i ! j !} \leq \max _{0 \leq i \leq M_{2} ; 0 \leq j \leq N_{2}} \frac{\left|f^{(i, j)}(z, w)\right|}{i ! j !}
$$

for all $i=0,1,2, \ldots ; j=0,1,2, \ldots ; k=0,1,2, \ldots$ and all $(z, w)$ such that $|z| \geq T,|w| \geq T$. Choosing $M \geq \max \left\{M_{1}, M_{2}\right\}$ and $N \geq \max \left\{N_{1}, N_{2}\right\}$, we have (1) for all $i=0,1,2, \ldots ; j=$ $0,1,2, \ldots$ and all $(z, w) \in \mathbb{C}^{2}$. 


\section{REFERENCES}

1. Krishna G.J., Shah S.M. Functions of bounded indices in one and several complex variables, Mathematical Essays Dedicated to A. J. Macintyre, Ohio University Press, Athens, Ohio, (1970), 223-235.

2. Bandura A.I., Skaskiv O.B., Analytic functions in the unit ball of bounded L-index: asymptotic and local properties, Mat. Stud., 48 (2017), №1, 37-73.

3. Bandura A., Skaskiv O., Functions analytic in a unit ball of bounded L-index in joint variables, J. Math. Sci., 227 (2017) №1, 1-12.

4. Bandura A., Skaskiv O., Directional logarithmic derivative and the distribution of zeros of an entire function of bounded L-index along the direction, Ukr. Math. J., 69 (2017), №3, 500-508.

5. Bordulyak M.T., Sheremeta M.M., Boundedness of l-index of analytic curves, Mat. Stud., 36 (2011), №2, 152-161.

6. Gross F., Entire Function of Bounded Index, Proc. Amer. Math. Soc., 18 (1967), 974-980.

7. Heath L.F., Vector-valued entire functions of bounded index satisfying a differential equation, Journal of Research of the NBS, 83B (1978), №1, 75-79.

8. Lepson B., Differential equations of infinite order, hyperdirichlet series and entire functions of bounded index, Proc. Sympos. Pure Math., 11 (1968), 298-307.

9. Nuray F., Patterson R.F., Entire bivariate functions of exponential type, Bulletin of Mathematical Sciences, 5 (2015), №2, 171-177.

10. Nuray F., Patterson R.F., Multivalence of bivariate functions of bounded index, Le Matematiche, 70 (2015), №2, 225-233.

11. Patterson R.F., Nuray F., A characterization of holomorphic bivariate functions of bounded index, Mathematica Slovaca, 67 (2017), №3, 731-736.

12. Roy R., Shah S.M., Growth properties of vector entire functions satisfying differential equations, Indian J. Math., 28 (1986), №1, 25-35.

13. Roy R., Shah S.M., Vector-valued entire functions satisfying a differential equation, J. Math. Anal. Appl., 116 (1986), №2, 349-362.

14. Salmassi M., Some classes of entire functions of exponential type in one and several complex variables, Doctoral dissertation, University of Kentucky, Lexington, Kentucky, 1978.

15. Salmassi M., Functions of bounded indices in several variables, Indian J. Math., 31 (1989) №3, 249-257.

16. Sheremeta M., Boundedness of $l-M$-index of analytic curves, Visn. Lviv Un-ty. Ser. Mech.-Math., 75 (2011), 226-231. (in Ukrainian)

Department of Mathematics, Afyon Kocatepe University

Afyonkarahisar, Turkey

fnuray@aku.edu.tr

Department of Mathematics and Statistics

University of North Florida Jacksonville, Florida

rpatters@unf.edu 